\title{
Aberrations of the Cervical Carotid Artery Which May Be Dangerous in Pharyngeal Surgery-A Computed Tomographic Study
}

\author{
Johannes Gossner ${ }^{1,2}$, Ricarda Manka ${ }^{1,3}$, Joerg Larsen ${ }^{1,2}$ \\ ${ }^{1}$ Institute for Roentgendiagnostics, Braunschweig Teaching Hospitals, Braunschweig, Germany \\ ${ }^{2}$ Department of Clinical Radiology, Weende Teaching Hospital, Göttingen, Germany \\ ${ }^{3}$ Euradia, Private Radiology Practice, Braunschweig, Germany \\ Email: johannesgossner@gmx.de
}

Received November 14, 2012; revised December 21, 2012; accepted January 4, 2013

\begin{abstract}
Background: There are recognised variations in the anatomical course of the cervical portion of the internal carotid artery. An aberrant vessel with direct contact to the pharyngeal wall could easily be injured during pharyngeal surgery or may appear as a pharyngeal pseudo mass. Previous anatomical studies predominantly involved older patients. The prevalence of such variations which are at risk of injury during pharyngeal surgery has thus not been established in a general patient population. Material and Methods: The course of the internal carotid artery in relation to the oro and hypopharyngeal walls was retrospectively evaluated bilaterally by simple visual inspection and measurement of the smallest distance between the respective vessels and the adjacent mucosal surface of the pharyngeal wall in 138 consecutive contrast-enhanced computed tomography scans of the neck. Results: 11/138 (7.9\%) of patients demonstrated relevant cervical carotid artery aberrations, comprising medial kinking of a vessel with asymmetry of the adjacent pharyngeal lumen and/or an intimate submucous course in the pharyngeal wall with no identifiable separating fat plane. This prevalence increased with age. Simple visual inspection correlated well with the measurement of the smallest distance between an artery and the pharyngeal wall, which was statistically significant $(p<0.0001)$. Conclusions: The prevalence of about $8 \%$ in a general patient population is higher than previously recorded in anatomical studies. Prevalence increases with age. Otorhinolaryngologists should be aware of such variation as a risk factor for haemorrhagic complications during pharyngeal surgery and as a differential diagnosis of pharyngeal mass lesions, especially in older patients. Modern contrast-enhanced CT allows identification and characterisation of any surgically relevant variant vascular anatomy in the pre-operative work-up.
\end{abstract}

Keywords: Computed Tomography; Internal Carotid Artery; Pharyngeal Surgery; Variant Anatomy

\section{Introduction}

Variations in the anatomical course of the cervical portion of the internal carotid artery (ICA) are frequent and reported to occur in about $6 \%-30 \%$ of patients with some authors even reporting a prevalence as high as $62 \%$ (as reviewed by Ovchinnikov et al.) [1]. In most cases, these are incidental findings lacking clinical relevance. However, an aberrant ICA showing direct contact to the pharyngeal wall could easily be injured during surgical procedures, e.g., pharyngeal abscess drainage or tonsillectomy [2]. Equally, it may appear as a mass lesion of the pharynx, sometimes associated with clinical signs such as dysphagia or globus syndrome [3-7]. For an aberrant cervical ICA which is at risk at surgery and/or causing a pseudomass appearance, the term "dangerous loop" has been proposed [8]. The previously performed anatomical and angiographic studies mostly reported on the anatomical variation per se, with some anatomical studies recording the prevalence of the so called "dangerous loop". Anatomical studies on human cadavers have the shortcoming of selection bias and naturally involve older patients. The prevalence of clinically relevant aberrations in a more general population is thus unclear. Cross-sectional imaging offers an opportunity to study human anatomy in vivo. With the use of intravenous contrast medium, the ICA can be easily identified and its topographical relations studied. Such studies are part of the routine investigations in almost any pre-surgical setting. The present study has investigated the prevalence of potentially dangerous variations of the cervical internal carotid artery in a general patient population of a large teaching hospital. 


\section{Material and Methods}

Retrospective study of contrast-enhanced computed tomography (CT) scans of the neck performed between October 2007 and February 2008 at the Institute of Roentgendiagnostics at Braunschweig Teaching Hospitals. According to the statute of the ethics of comittee of the affiliated Hannover medical school (Medizinische Hochschule) retrospective studies of available data are possible without dedicated statement. Patients were identified from the departmental RIS-data base and considered for inclusion into the study $(n=236)$. Patients with mass lesions of the oro- or hypopharynx (such as abscesses or malignancies) causing luminal asymmetry, any history of cervical surgery (specifically neck dissection), thrombosis of an ICA as well as intubated patients were excluded from the analysis. Scans with excessive beam hardening artefacts due to metallic dental implants were also excluded. A total of 138 examinations were finally included. The study population consisted of 91 men and 47 women (1.9:1) with an average age of 59.1 years (17 86 years). The 98 excluded patients had similar base-line characteristics considering gender and mean age. Most patients included in the study were being staged for various malignancies (lymphoma, breast cancer, melanoma, head and neck tumours) or were examined to exclude a clinical suspected parapharyngeal abscess.

A 16-slice CT scanner (Somatom ${ }^{\circledR}$, Siemens Medical Solutions, Germany) and an 8-slice CT scanner (LightSpeed $^{\circledR}$, GE Healthcare, USA) were used. Scanning was performed from the orbital floor to the upper thoracic aperture at a slice thickness of $2 \mathrm{~mm}$ with the Siemens and $1.25 \mathrm{~mm}$ with the GE-Scanner. $120 \mathrm{~mL}$ of non-ionic iodinated contrast media (Ultravist ${ }^{\circledR}$, Bayer-Schering, Germany) was administered to each patient at a flow-rate of 2 - $3 \mathrm{~mL} / \mathrm{s}$. For better arterial enhancement, a twophased acquisition was performed in most patients according to standard departmental protocol: the contrast medium volume was split into two portions $(2 \times 60 \mathrm{~mL})$, the second portion being administered 40 seconds following the first portion with scanning being started 60 seconds after the first injection of contrast medium. In the remainder, a fixed delay of 60 seconds following injection of contrast medium was used.

All scans were reviewed on transverse-axial reconstructions in soft tissue window settings (WL 40 - 50, WW 350) at a standard medical post-processing workstation. CT studies were initially evaluated by simple visual inspection by a single observer who routinely reports independently on cervical CT scans (JG). Any variation in the course of an internal carotid artery was judged as potentially dangerous if it caused any asymmetry of the oro or hypopharyngeal lumen and/or if the ICA showed a direct sub-mucous course with no identifiable separating fat plane to the pharyngeal wall. In patients with a relevant anatomic variation, thin section multiplanar reformations were performed for better depiction of the topographic anatomy. To test the reliability of the simple visual inspection assessment, the smallest distance between the internal carotids and the pharyngeal lumen was finally measured in all patients by a second examiner (RS). A cut-off value of $\leq 5 \mathrm{~mm}$ was chosen. The accordance between patient with visually judged "dangerous loop" and patients with a measured smallest distance between the pharyngeal wall and the internal carotids $\leq 5 \mathrm{~mm}$ was verified. The measured distances between the grop with and without visually rated "dangerous loop" were also compared using the t-test. For a signifiant difference a $\mathrm{p}$-value $<0.05$ was defined. For the calculation Statistica ${ }^{\circledR}$ was used (Version 5.5, ' 99 Edition, StatSoft, USA).

\section{Results}

11 of 138 patients $(7.9 \%)$ showed potentially dangerous variations in the course of the cervical ICA, i.e. an asymmetry of the lumen of the hypo- or oro-pharyngeal lumen and/or a direct sub-mucous course of the vessel with no identifiable separating fat plane to the adjacent pharyngeal wall (Figure 1). Their average age was 73.6 years (range 54 - 86 years) of whom 7 were men and 4 were women. One patient showed a sub-mucous course of the carotid artery in isolation, while the luminal asymmetry was only slight in two others. Prevalence increased from $2 \%(2 / 99)$ in patients younger than 70 years to $23.6 \%(9 / 38)$ in patients aged 70 or older.

All measurements in patients showing a potentially dangerous variant were $\leq 5 \mathrm{~mm}(100 \%)$. Of the patients

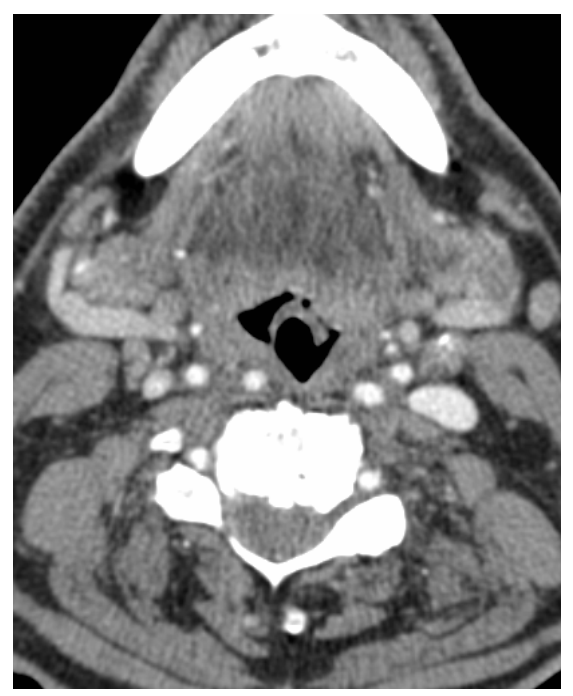

Figure 1. Axial CT image of a 75-year-old man undergoing staging of suspected cancer of the hard palate. There is medial elongation of the right internal carotid artery associated with luminal asymmetry, the so called "dangerous loop”. 
with no obvious dangerous arterial variation on visual inspection, only $0.4 \%$ of all distance measurements were $\leq 5 \mathrm{~mm}$. The mean distances between the two groups were statistically significant $(\mathrm{p}<0.0001)$.

\section{Discussion}

This study has investigated the occurrence of potentially dangerous variations in the course of the cervical internal carotid artery in a general patient population using contrast-enhanced cervical CT scans. Because surgical procedures are routinely performed in this area, such anatomical variations are an obvious concern: A close contact between an artery and the oro- or hypopharyngeal wall may become a critical haemorrhagic risk at the time of surgical interventions. For example, injury to an ICA during tonsillectomy may result in life threatening bleeding [2]. On the other hand, these aberrations are an important differential diagnosis in the evaluation of pharyngeal masses such as tumorous entities or abscesses and any recognised variant anatomy may clearly alter patient management [2-8]. The differentiation of clinical relevant and irrelevant variations is therefore of considerable value, especially as relevant variations are described in as much as $62 \%$ of patients [1]. It appears that some elongation or kinking of the cranial portions of the cervical segments of the carotid arteries is a normal compensatory mechanism to prevent overstretching and narrowing during movement of this important blood supply to the brain [1]. Prevalences of anatomic variants recorded in the literature are wide ranging from 5.3\% to $62.4 \%$ (with $n=1123$ [9], and $n=590$ [10], respectively). This may be explained by the use of different methods of assessment, namely cadaveric, angiographic or ultrasonographic studies in addition to different definitions of what may constitute a clinically relevant variation. No study has reported on findings from cross sectional imaging data. Finally, it is noteworthy that anatomical variants as considered here may also be critically important during neuroendovascular interventions such as stentprotected carotid angioplasty or the coilembolisation of intracranial aneurysms [11], however, these aspects are beyond the scope of this study, which has focused solely on potentially dangerous variants in the context of pharyngeal surgery.

Few studies have considered surgically relevant variations of ICA anatomy, specifically a small distance between the ICA and the adjacent pharyngeal wall. Tillmann and Christofides were first to report a prevalence of $2.2 \%(2 / 89)$ in their anatomic study and proposed the term "dangerous loop" [8]. Paulsen et al. studied the course of the ICA in human cadavers. Kinking or coiling with direct relation to the pharyngeal wall was noted in $2.8 \%(8 / 282)$ [12]. In another anatomical study by Ozgur et al., 4\% (2/50) of cases showed a direct relation between artery and the pharyngeal wall [13]. In our study, we found a higher prevalence of $7.9 \%$. This may in part be explained by the different methodology: When using fixation with formalin on human cadavers, some shrinking of the tissue occurs which may alter parapharyngeal topographic anatomy [14]. In contrast, cross-sectional imaging allows the examination of human anatomy in vivo. In a very recent study Jun et al. found a distance of less than $5 \mathrm{~mm}$ between the internal carotid artery and the hypopharyngeal wall in around $10 \%$ patients, which is in accordance to our data. But they did not record morphologic features of the "dangerous loop" like luminal assymmetry or direct submucous course of the artery, which is in our opinion a clinical relevant aspect [15]. In addition, we have also excluded scans of patients in whom pharyngeal anatomy was disturbed due to malignant or inflammatory masses, intubation or postoperative changes in order for observed pharyngeal asymmetry being solely attributable to an aberrant course of an ICA. Some overdiagnosis of patients showing luminal asymmetry could have occurred because asymmetry may also be observed during deglutition. However, the verification of a direct submucous and thereby potentially dangerous course of an ICA is independent of swallowing.

Where an ICA had an intimate relation to the adjacent pharyngeal wall, the vessel was elongated and kinked medially in all cases. The aetiology of this elongation and kinking is not clear. Generally it is believed that looping or coiling is due to congenital changes, whereas elongation with kinking is acquired and ascribed to atherosclerosis: a recent histological study of such a cohort described metaplastic changes of the ICA with a reduction in elastic fibres and muscular cells, making the vessel susceptible to haemodynamic forces such as in arterial hypertension [16]. Elongation and unfolding of the thoracic aorta have of course long been believed to be due to long-standing hypertension [17]. More recently, this observation has also been made for the carotid arteries [10]. In addition, recently, Lam et al. reported an increased prevalence of tortuosity of the common and internal carotid arteries with increasing age in their angiographic study of the vascular anatomy in patients undergoing carotid angioplasty and stenting [11]. Progressive kinking in relation to ageing has also been demonstrated for the femoral artery [18]. In our sample, no case of potentially dangerous elongation and kinking was found in patients younger than 50 years and rarely occurred in patients between 50 and 70 years of age, as would be expected with an acquired condition.

The mean age of our patients was 59.1 years, with the youngest being 17 years old. Our data can thus not be easily applied to children, who commonly undergo pharyngeal surgery, especially tonsillectomy. It is notewor- 
thy therefore that Galetti et al. reported on an 8-year-old child who complained of dysphagia and pharyngodynia and was found to have kinking of the right ICA, giving rise to an impression on the adjacent pharyngeal wall [2]. A possible explanation arises from the embryologic development of the ICA from brachial arch arteries: a persisting loop of the ICA could therefore be interpreted as a rudimentary stage of development $[11,12]$. There are also rare congenital syndromes with tortuosity of the great vessels. However, these are usually accompanied by other clinical stigmata such as skin and joint laxity in Marfan's syndrome [19], which should prompt further consideration of vascular anomalies. As indicated above, the published anatomical studies involved predominantly older patients, so that no conclusions could be drawn for younger patients. Nonetheless, the fact that no clinically relevant anatomic variant of the ICA could be observed in patients under the age of 50 years in our example as well as the evidence for an acquired aetiology as cited above makes a high prevalence of potentially dangerous variations in children unlikely.

In our cohort, cervical CT scans with contrast enhancement demonstrated adequate depiction of carotid anatomy in relation to the pharyngeal wall, similar to previous reports [4-7]. CT imaging is a widely available, robust, fast and accurate method of imaging carotid vessel anatomy. The possibility of acquiring thin slices offers the additional opportunity of high quality multiplanar reformations. There are no contraindications like pacemakers and even patients with claustrophobia are usually examinable with CT. CT scans obtained for diagnostic purposes and pre-surgical planning can readily be used for the study of the vascular anatomy. For better depiction of the vascular anatomy and to obtain sufficient parenchymal contrast, a split-bolus technique was used in most patients in our study, a technique first described by Platt and Gazer for abdominal imaging [20]. However, in our experience, even a delayed enhancement provides sufficient contrast to depict gross vascular pathology. Nonetheless, there are several other imaging approaches to demonstrate cervical vascular anatomy. MRI has the advantage of lacking the use of ionising radiation, which is particularly important when imaging children. Besides from being generally less available, MRI has the shortcoming of longer scan times with more artefacts occurring because of movement, specifically from respiration and swallowing. MRI also commonly uses slice thicknesses in the $3-5 \mathrm{~mm}$ range, making the study of detailed parapharyngeal anatomy problematic, and additional sequences such as MR-angiography may often be required for full assessment. Especially older patient frequently have contraindications like pacemakers. An ultrasonographic examination has the advantage of being an easy to use bed-side technique which allows the study of the course of the ICA and possible variations thereof [21]. In our opinion, ultrasound could easily be used as a screening test, while cross-sectional imaging offers superior demonstration of the detailed topographical anatomy which is needed for surgical planning. In contrast, conventional angiography, apart from being an invasive technique which carries the risk of occasionally deleterious consequences such as peri-procedural stroke, is not a good choice for evaluating anomalies of the carotid arteries since it neither shows the surrounding soft tissues nor the relation of a vessel to the pharyngeal wall.

The simple visual judgement of ICA anatomy initially applied in this study showed a good correlation with the more objective measurement of the minimal distance between the ICA and the pharyngeal lumen. All measurements in patients showing a potentially dangerous variation were $\leq 5 \mathrm{~mm}$, in patients with no obvious clinical variation no more than $0.4 \%$ had such a narrow relation. The measurements in the two groups were statistically significant $(p<0.0001)$. One might argue that only the measured proximity between artery and mucosa is a sufficient indicator for a dangerous aberration, however, in everyday practice, any measurements are time consuming and checking for an asymmetry appears to us to be a fair compromise when aiming to detect clinically relevant carotid aberrations. In fact, all of the anatomical studies cited above have solely used simple visual inspection and description of ICA anatomy following dissection since this is the standard method in macroscopic anatomy [8-12]. Nonetheless, the judgement of topographical relations depends on the view point of the anatomist and exact measurements of any alteration in anatomy are clearly not easy to perform. These limitations are overcome by the use of cross-sectional imaging where multi-planar assessment of volume data sets as routinely acquired in CT scanning is compulsory [22]. The patient sample was selected for the study of the prevalence of potential dangerous variant anatomy of the internal carotid artery. Patients with pathology disturbing normal anatomy where excluded. So, in this sample there was no direct impact on patient management. But, as a consequence of this study the radiologists of the authors department were sensitized of such dangerous variant anatomy and we started to routinely comment on possible dangerous arterial variant anatomy in cervical CTscans.

In conclusion, potentially dangerous variant anatomy of an internal carotid artery is seen in about $8 \%$ of cases in a general patient population undergoing cervical CT scanning. This prevalence is higher than that which has been reported in anatomical studies previously. Prevalence increases with age in accordance with the proposed degenerative aetiology. Otorhinolaryngologists and head and neck surgeons should be aware of these variations as 
a risk factor for major haemorrhage during pharyngeal surgery and as a differential diagnosis in the consideration of pharyngeal masses, specifically in the elderly. Modern contrast-enhanced CT allows the identification and characterisation of any surgically relevant variant vascular anatomy in the pre-operative work-up, even if delayed enhancement protocols are being used. Equally, radiological reports on cervical CT scans should routinely comment on any such variant arterial anatomy.

\section{Acknowledgements}

An abstract of this study will presented as a poster at the European Congress of Radiology in March 2013 at Vienna.

\section{REFERENCES}

[1] N. A. Ovchinnikov, R. T. Rao and R. R. Rao, "Unilateral Congenital Elongation of the Cervical Part of the Internal Carotid Artery with Kinking and Looping: Two Case Reports and Review of the Literature," Head \& Face Medicine, Vol. 3, 2007, p. 26.

http://www.head-face-med.com/content/3/1/29

[2] B. Galletti, S. Bucolo, G. Abbate, et al., "Internal Carotid Transposition as Risk Factor in Pharyngeal Surgery," Laryngoscope, Vol. 112, No. 10, 2002, pp. 1845-1848. doi:10.1097/00005537-200210000-00026

[3] M. Jäckel, "Variations in the Clinical Course of the Internal Carotid Artery-A Differential Diagnosis of Parapharyngeal Masses," HNO, Vol. 45, No. 12, 2007, pp. 1018-1021.

[4] J. N. Lin, T. C. Tsai, C. H. Lin and F. C. Hsieh, "A Dangerous Variant Causing Retropharyngeal Space Enlargement, Retropharyngeal Internal Carotid Artery: A Case Report," Chinese Journal of Radiology, Vol. 32, No. 1, 2007, pp. 27-30.

[5] F. Calzolari and A. Salett, "Retropharyngeal Internal Carotid Artery. Diagnosis by CT Angiography in 5 Cases," Radiologia Medica, Vol. 95, No. 4, 1998, pp. 383-385.

[6] J. Vega, C. Gervas, G. Vega-Hazas, et al., "Internal Carotid Artery Transposition: Another Cause of Widening of the Retropharyngeal Space," European Radiology, Vol. 9, No. 2, 1999, pp. 347-348. doi:10.1007/s003300050678

[7] E. Aydin, G. Akkuzu, B. Akkuzu and L. N. Özlüoglu, "Tortuous Internal Carotid Artery Indenting the Piriform Sinus: A Case Report," European Archives of Oto-RhinoLaryngology, Vol. 262, No. 5, 2005, pp. 351-352.

[8] B. Tillmann, C. Christofides, "The 'Dangerous Loop' of the Internal Carotid Artery. An Anatomic Study," HNO, Vol. 43, No. 10, 1995, pp. 601-604.

[9] G. E. Poulias, B. Skoutas, N. Doundoulakis, et al., "Kinking and Coiling of Internal Carotid Artery with and without Associated Stenosis. Surgical Considerations and LongTerm Follow-Up," Panminerva Medica, Vol. 38, No. 1, 1996, pp. 22-27.

[10] P. Pancera, M. Ribul, B. Presciutti and A. Lechi, "Preva- lence of Carotid Artery Kinking in 590 Consecutive Subjects Evaluated by Echocolordoppler. Is There a Correlation with Arterial Hypertension?" Journal of Internal Medicine, Vol. 248, No. 1, 2000, pp. 7-12. doi:10.1046/j.1365-2796.2000.00611.x

[11] R. C. Lam, S. C. Lin, B. DeRubertis, et al., "The Impact of Increasing Age on Anatomic Factors Affecting Carotid Angioplasty and Stenting," Journal of Vascular Surgery, Vol. 45, No. 5, 2007, pp. 875-880.

doi:10.1016/j.jvs.2006.12.059

[12] F. Paulsen, B. Tillmann, C. Christofides, et al., "Curving and Looping of the Internal Carotid Artery in Relation to the Pharynx: Frequency, Embryology and Clinical Implications," Journal of Anatomy, Vol. 197, No. 3, 2000, pp. 373-381. doi:10.1046/j.1469-7580.2000.19730373.x

[13] Z. Ozgur, S. Celik, F. Govsa, et al., "A Study of the Internal Carotid Artery in the Parapharyngeal Space and Its Clinical Importance," European Archives of Oto-RhinoLaryngology, Vol. 264, No. 12, 2007, pp. 1483-1489. doi:10.1007/s00405-007-0398-6

[14] W. Steinmann, "Makroskopische Präperationsmethoden in der Medizin," Thieme Publishers, Stuttgart, 1982.

[15] B. C. Jun, E. J. Jeon, D. H. Kim, et al., "Risk Factors of Decreased Distance between Internal Carotid Artery and Pharyngeal Wall," Auris Nasus Larynx, Vol. 39, No. 6, 2012, pp. 615-619. doi:10.1016/j.anl.2011.10.018

[16] G. La Barbera, G. La Marca, A. Martino, et al., "Kinking, Coiling, and Tortuosity of Extracranial Internal Carotid Artery: Is It the Effect of Metaplasia?" Surgical and Radiologic Anatomy, Vol. 28, No. 6, 2006, pp. 573-580. doi:10.1007/s00276-006-0149-1

[17] I. Steinberg, "The Arteriosclerotic Aorta: Clinical and Roentgen Observations," Angiology, Vol. 7, No. 5, 1956, pp. 405-418.

[18] P. J. W. Wensing, F. G. Scholten, P. C. Buijs, et al., “Arterial Tortuosity in the Femoropoliteal Region during Flexion: An MRA Study," Journal of Anatomy, Vol. 187, No. 1, 1995, pp. 133-139.

[19] P. Franceschini, A. Guala, D. Licata, et al., "Arterial Tortuosity Syndrome," American Journal of Medical Genetics, Vol. 91, No. 2, 2000, pp. 141-143. doi:10.1002/(SICI) 1096-8628(20000313)91:2<141::AIDAJMG13>3.0.CO;2-6

[20] J. F. Platt and G. M. Glazer, "IV Contrast Material for Abdominal CT: Comparison of Three Methods of Administration," American Journal of Roentgenology, Vol. 51, No. 2, 1998, pp. 275-277.

[21] L. Pellegrino, G. Prencipe and F. Vairo, "Dolicho-Arteropathies (Kinking, Coiling, Tortuosity) of the Carotid Arteries: Study by Color Doppler Ultrasonography," Minerva Cardioangiology, Vol. 46, No. 1-2, 1998, pp. 6976.

[22] R. Corti, M. Alerci, R. Wyttenbach, et al., "Usefulness of Multiplanar Reconstructions in Evaluation of Carotid CT Angiography," Radiology, Vol. 226, No. 1, 2003, pp. 290291. doi:10.1148/radiol.2261020548 